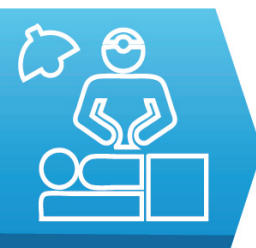

SURGERY

\title{
Desmoid tumor of the mesentery. Case report of a rare non-metastatic neoplasm
}

Miana Pop ${ }^{1}$, Dana Bartos ${ }^{1,2}$, Ofelia Anton ${ }^{3}$, Ioana Rusu ${ }^{4}$, Lidia Ciobanu ${ }^{5}$, Caius Breazu ${ }^{6,7}$, Ioana Iancu ${ }^{1,2}$, Cornel Iancu ${ }^{2,8}$, Adrian Bartos ${ }^{2,8}$

1) Department of Anatomy and Embryology, Iuliu Hatieganu University of Medicine and Pharmacy, Cluj-Napoca, Romania

2) Department of Surgery, Regional Institute of Gastroenterology and Hepatology, Cluj-Napoca, Romania

3) Department of Radiology, Regional Institute of Gastroenterology and Hepatology, Cluj-Napoca, Romania

4) Department of Anatomical Pathology, Regional Institute of Gastroenterology and Hepatology, Cluj-Napoca, Romania

5) Department of Gastroenterology, Regional Institute of Gastroenterology and Hepatology, Cluj-Napoca, Romania

6) Department of Anesthesiology and Intensive Care, Regional Institute of Gastroenterology and Hepatology, Cluj-Napoca, Romania

7) Department of Anesthesia, Iuliu Hatieganu University of Medicine and Pharmacy, Cluj-Napoca, Romania

8) Department of Surgery, Iuliu Hatieganu University of Medicine and Pharmacy, Cluj-Napoca, Romania

DOI: $10.15386 / \mathrm{mpr}-1620$

Manuscript received: 05.03.2020

Received in revised form: 27.01.2021

Accepted: 13.02.2021

Address for correspondence:

bartosdanamonica@gmail.com

This work is licensed under a Creative Commons Attribution-NonCommercialNoDerivatives 4.0 International License

\begin{abstract}
Desmoid tumors (DT) are rare non-metastatic neoplasms that occur through myofibroblast proliferation in musculoaponeurotic or fascial structures of the body, being commonly diagnosed in young women during pregnancy or in the post-partum period. We present the case of a 38-year-old woman, who recently gave birth, manifesting non-specific abdominal symptoms. Computed tomography indicated the presence of a solitary tumor arising from the intestinal wall or from the mesentery. Surgery confirmed the diagnosis, revealing a tumor that was localized at the level of the jejunal mesentery, having about $7 \mathrm{~cm}$ in diameter, in tight contact with the duodenum and the mesenteric vessels. "En bloc" resection of the tumor was performed, together with the involved enteral loops followed by end-to-end anastomosis of the jejunum. Histopathological examination of the surgical specimen sustained the diagnosis of desmoid tumor.
\end{abstract}

Keywords: desmoid tumors, jejunal mesentery, postpartum, surgical resection

\section{Introduction}

Desmoid-type fibromatosis or desmoid tumors (DT) are rare nonmetastatic neoplasms that occur through myofibroblast proliferation in musculoaponeurotic or fascial structures of the body. The incidence of DT in the general population is $2-4 /$ million/ year [1]. DT are more commonly diagnosed in younger women during pregnancy or post-partum period. DT can present as sporadic tumors, but in some cases, they can be associated with familial adenomatous polyposis (FAP) or with Gardner's syndrome [3]. Although histologically benign, DT are characterized by local invasiveness, especially in the mesentery, and high risk of recurrence after surgery [4].

\section{Case Report}

A 38-year-old woman, with no significant medical history, who recently gave birth, presented with acute onset of diffuse abdominal pain, asthenia and loss of appetite for the past 2 weeks. She described the suffering as a continuous pain in the entire abdomen, with back radiation and intermittent episodes of severe pain. Blood tests revealed anemia $(\mathrm{Hb} 8.6 \mathrm{~g} / \mathrm{dl})$ and thrombocytosis (791.000/ microL).

Computed tomography scan of the abdomen and pelvis was performed indicating a solitary tumor arising from the intestinal wall or from the mesentery, measuring 5/7/6 cm (Figure 1). The lesion had a pedicle with a $9 \mathrm{~mm}$ base of implantation and two aeric inclusions inside. Lymphadenopathy was observed in the perilesional fatty tissue. No displacement of the surrounding vessels was described (only a tight contact).

Intraoperatively, the mass was localized at the level of the jejunal mesentery, having about 7-8 $\mathrm{cm}$ in diameter, in tight contact with the duodenum and the mesenteric vessels (Figure 2a). "En bloc" resection of the tumor was performed, together with the involved enteral loops (about $40 \mathrm{~cm}$ of enteral loops were resected) (Figure 2b), followed by endto-end anastomosis of the jejunum. The patient recovered well and was discharged in the $6^{\text {th }}$ post-operative day. 


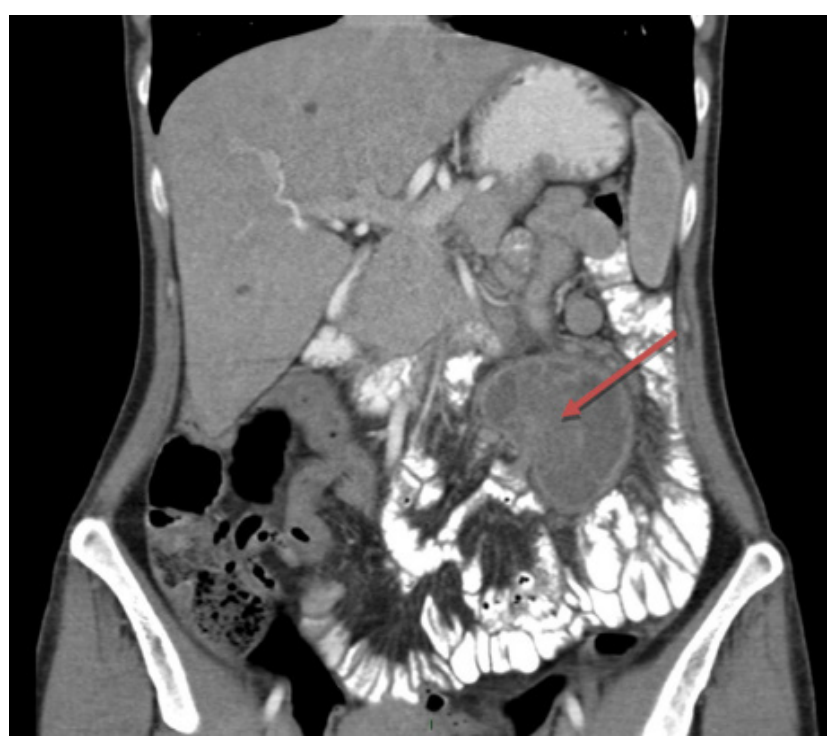

Figure 1. Computed tomography of the abdomen showing the intestinal lesion (red arrow).
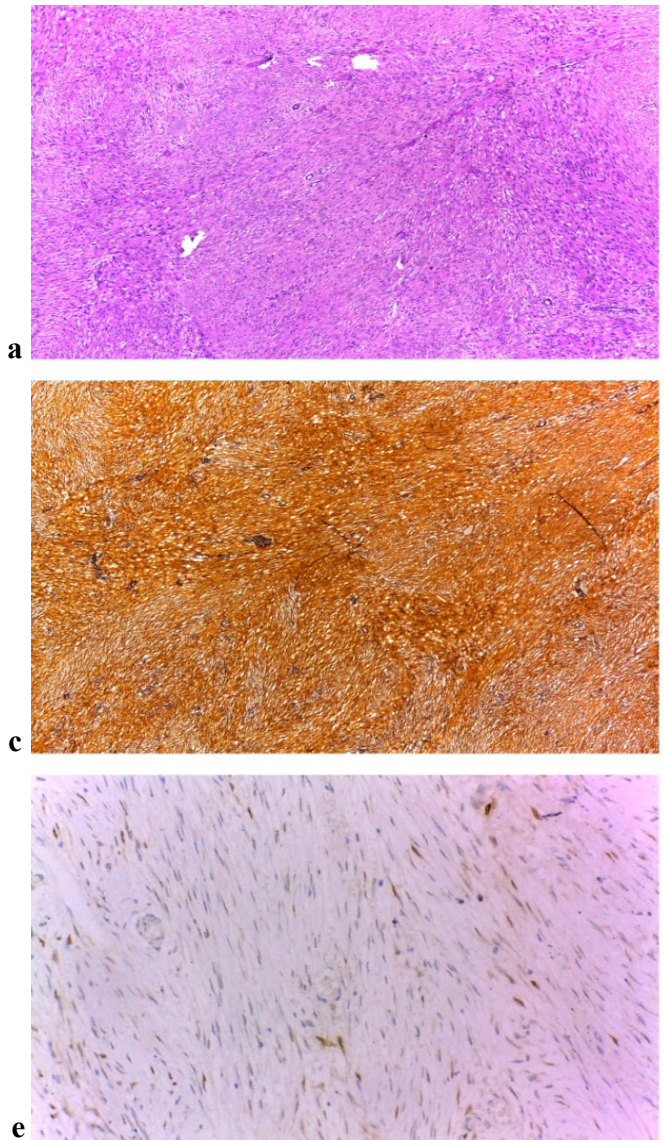

Figure 2. a. "In situ" intraoperative aspect (upper image).

b. Surgical piece after resection (lower image).
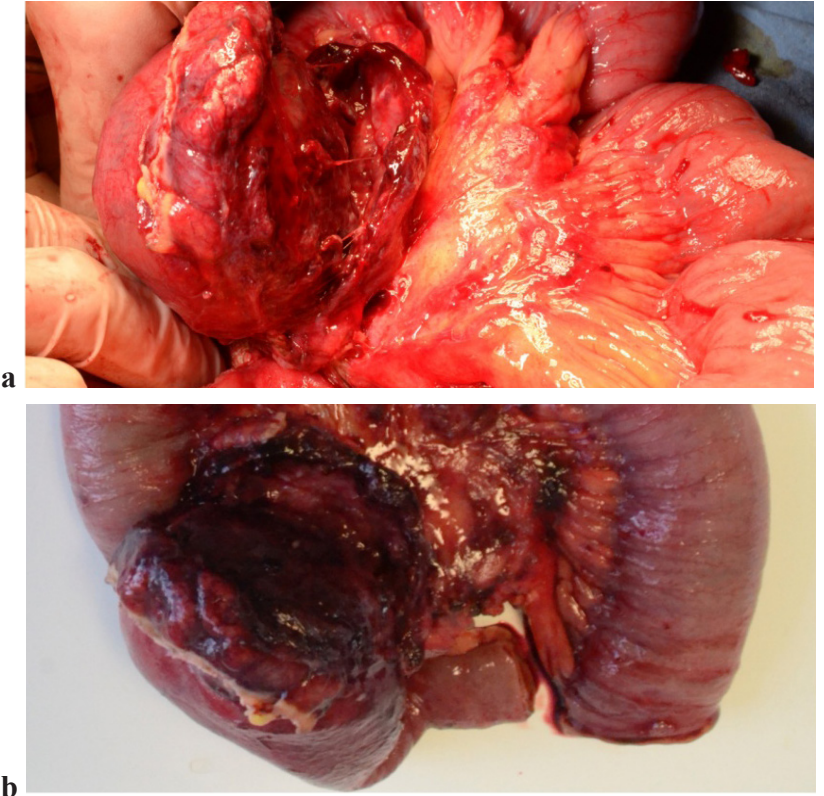
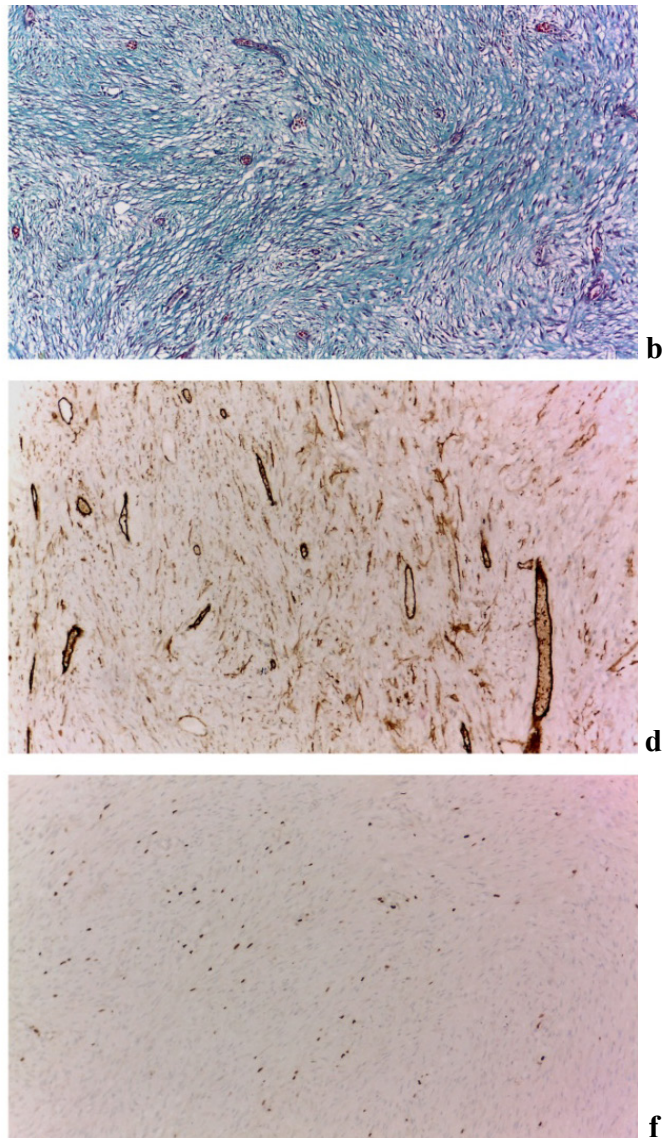

Figure 3. a. HE staining 4X. Fusocelular proliferation; b. Tricrom-Manson staining 10X. Green coloration of fibrocytic-fibroblast proliferation; c. Gomori 10X; d. CD34 10X. Fuzocelulare positive focal proliferation marker (and the vascular endothelial cells); e. Beta-Catenin 20X. Intracytoplasmic focal marker; f. 10X Ki67 proliferation index 10\%. 
Histopathological examination of the surgical specimen revealed a 7/6/5.5 $\mathrm{cm}$ tumor. The circumferential margin was microscopically positive (R1) with the other margins free of tumor (R0). Microscopical examination showed a moderate proliferation of spindle and few stellate cells, with indistinct cell boundaries and elongated nuclei arranged in intersecting fascicles with collagen fibers interspersed. The lesion had infiltrative margins including the submucosa of the small bowel. In the periphery of the lesion, recent bleeding, granulation tissue and fibrinhematic deposits were seen, along with a mixt inflammatory process. Mitotic index was $3 / 10$ and no intratumoral necrosis was highlighted. Immunohistochemistry was made with negative results for SMA, c-Kit, DOG1, S100, $\mathrm{CD} 34$. B-catenin was stained positive and the proliferation index Ki-67 was $10 \%$, all of this sustaining the diagnosis of desmoid tumor (Figure 3a-f).

No postoperative chemotherapy was considered as necessary at the oncological evaluation.

Currently, after 6 months since the surgery, clinical examination and imaging control offers normal relations, with no signs of recurrence.

\section{Discussion}

Desmoid tumors are benign lesions consisting of fibromatous tissue. They occur due to a defect in collagen formation [5]. DT can be classified in intra- or extraabdominal lesions (shoulder, thigh). Small bowel mesentery is the most common localization [6]. Although DT can be associated with polyposis syndromes, they are more often presented as solitary tumors. The frequency of DT is higher in young women during or after pregnancy and is closely correlated with estrogen levels. A higher incidence of the disease is found after use of oral contraceptives or at menopause [7]. DT diagnosed during pregnancy period are characterized by slow development and attenuated symptoms [8]. Also, in the case presented, the patient had recently given birth.

Intra-abdominal desmoid tumors are characterized by heterogeneous clinical manifestations. The most common symptom in DT is the abdominal pain associated with bowel disorders. DT can also evolve as asymptomatic lesions but more often the clinical picture is determined by the invasion of the surrounding structures. In our case, the patient's major complaint was linked to an abdominal discomfort along with recent periods of constipation.

$\mathrm{CT}$ or MRI scans are required for the diagnosis of DT. The heterogeneous signal that is often found varies depending on the number of fibroblasts that proliferate, the vascularity of the tumor and the presence of collagen tissue in the tumor [9]. Most DT appear as well-defined, homogenous, isodense or hyperdense structures in relation with the adjacent muscles [10]. In our case, abdominal CT scan with contrast enhancement confirmed the nonspecific radiologic picture of this tumor, with some minor discrepancy between CT imaging and intraoperative findings.

The role of MR examination in DT imaging consists in the capacity of delineation between tumor and local invasion with better accuracy [11].

The treatment of DT can be surgical or nonsurgical. Non-surgical management options of DT include observation, radiotherapy, systemic chemotherapy, hormonal substitutes, antiestrogen therapy. Until recently, complete excision of the tumor with negative margins was the gold standard therapy [12]. Because the infiltrative nature of this disease and the frequently evidenced invasion into different structures, this goal was increasingly difficult to achieve. Further studies concluded that positive margins were not a negative prognostic factor in the patient's followup and that function-sparing surgery should be preferred to aggressive surgery seeking negative margins $[13,14]$. The role of radiotherapy after surgery with positive margins is controversial and the observation of these patients is considered to be the best approach [15].

Given the high risk of local recurrence after surgery and the possible activation of B-catenin pathway by the growth factors released during wound healing process, non-surgical treatment options were also considered for patients with DT [16]. Antiestrogen agents (tamoxifen, toremifene) alone or in combination with anti-COX2 drugs (indomethacin or sulindac) were found to positively influence DT patient evolution. Chemotherapy with methotrexate and vinblastine or doxorubicin should be used only for cases with previous treatment failure, considering the fact that DT are benign tumors and metastatic disseminations were not described [17]. Another controversial factor for the prognosis of the disease is Ki67 index, which seems to associate a higher risk of recurrence if positive. However, there is no threshold value that can predict the prognosis of desmoid tumors [18]. Comparing literature data with this case, Ki67 index was relatively not high and the 6 months follow-up did not show any signs of recurrence.

Unfortunately, given the rarity of this disease, no evidence-based approach for the treatment is available. A multidisciplinary approach of individualized patients is required for optimal therapeutic management.

\section{Consent}

For publication of this case report and any accompanying images, written informed consent was obtained from the patient.

\section{References}

1. McKinnon JG, Neifeld JP, Kay S, Parker GA, Foster WC, Lawrence W Jr. Management of desmoid tumors. Surg Gynecol Obstet. 1989;169:104-106.

2. de Bree E, Keus R, Melissas J, Tsiftsis D, van Coevorden 
F. Desmoid tumors: need for an individualized approach. Expert Rev Anticancer Ther. 2009;9:525-535.

3. Shinagare AB, Ramaiya NH, Jagannathan JP, Krajewski KM, Giardino AA, Butrynski JE, et al. A to $\mathrm{Z}$ of desmoid tumors. AJR Am J Roentgenol. 2011;197:W1008-W1014.

4. Smith AJ, Lewis JJ, Merchant NB, Leung DH, Woodruff JM, Brennan MF. Surgical management of intra-abdominal desmoid tumours. Br J Surg. 2000;87:608-613.

5. Häyry P, Reitamo JJ, Tötterman S, Hopfner-Hallikainen D, Sivula A. The desmoid tumor. II. Analysis of factors possibly contributing to the etiology and growth behavior. Am J Clin Pathol. 1982;77:674-680.

6. Burke AP, Sobin LH, Shekitka KM, Federspiel BH, Helwig EB. Intra-abdominal fibromatosis. A pathologic analysis of 130 tumors with comparison of clinical subgroups. Am J Surg Pathol. 1990;14:335-341.

7. Clark SK, Phillips RK. Desmoids in familial adenomatous polyposis. Br J Surg. 1996;83:1494-1504.

8. Church JM, McGannon E. Prior pregnancy ameliorates the course of intra-abdominal desmoid tumors in patients with familial adenomatous polyposis. Dis Colon Rectum. 2000;43:445-450.

9. Lee JK, Glazer HS. Controversy in the MR imaging of fibrosis. Radiology. 1990;177:21-22.

10. Einstein DM, Tagliabue JR, Desai RK. Abdominal desmoids: CT findings in 25 patients. AJR Am J Roentgenol. 1991;157:275-279.

11. Kushihashi T, Munechika H, Otsuki N, Sato S, Kubota H, Ri $\mathrm{K}$, et al. CT and MR imaging of desmoid tumors--comparison of two modalities. Nihon Igaku Hoshasen Gakkai Zasshi. 1993;53:526-534

12. Colombo C, Gronchi A. Desmoid-type fibromatosis: what works best? Eur J Cancer. 2009;45 Suppl 1:466-467.

13. Gronchi A, Casali PG, Mariani L, Lo Vullo S, Colecchia M, Lozza L, et al. Quality of surgery and outcome in extraabdominal aggressive fibromatosis: a series of patients surgically treated at a single institution. J Clin Oncol. 2003;21:1390-1397.

14. Salas S, Dufresne A, Bui B, Blay JY, Terrier P, RanchereVince D, et al. Prognostic factors influencing progressionfree survival determined from a series of sporadic desmoid tumors: a wait-and-see policy according to tumor presentation. J Clin Oncol. 2011;29:3553-3558.

15. Meazza C, Bisogno G, Gronchi A, Fiore M, Cecchetto G, Alaggio R, et al. Aggressive fibromatosis in children and adolescents: the Italian experience. Cancer 2010;116:233240 .

16. Cheon SS, Nadesan P, Poon R, Alman BA. Growth factors regulate beta-catenin-mediated TCF-dependent transcriptional activation in fibroblasts during the proliferative phase of wound healing. Exp Cell Res. 2004;293:267-274.

17. Briggs A, Rosenberg L, Buie JD, Rizvi H, Bertagnolli MM, Cho NL. Antitumor effects of hyaluronan inhibition in desmoid tumors. Carcinogenesis. 2015;36:272-279.

18. Lin XY, Wang L, Zhang Y, Dai SD, Wang EH. Variable Ki67 proliferative index in 65 cases of nodular fasciitis, compared with fibrosarcoma and fibromatosis. Diagn Pathol. $2013 ; 8: 50$ 